\title{
An algorithm based on logistic regression with data fusion in wireless sensor networks
}

\author{
Longgeng Liu*, Guangchun Luo', Ke Qin ${ }^{1}$ and Xiping Zhang ${ }^{2}$
}

\begin{abstract}
A decision fusion rule using the total number of detections reported by the local sensors for hypothesis testing and the total number of detections that report "1" to the fusion center (FC) is studied for a wireless sensor network (WSN) with distributed sensors. A logistic regression fusion rule (LRFR) is formulated. We propose the logistic regression fusion algorithm (LRFA), in which we train the coefficients of the LRFR, and then use the LRFR to make a global decision about the presence/absence of the target. Both the fixed and variable numbers of decisions received by the $F C$ are examined. The fusion rule of $K$ out of $N$ and the counting rule are compared with the LRFR. The LRFA does not depend on the signal model and the priori knowledge of the local sensors' detection probabilities and false alarm rate. The numerical simulations are conducted, and the results show that the LRFR improves the performance of the system with low computational complexity.
\end{abstract}

Keywords: Logistic regression, Data fusion, Wireless sensor network, Counting rule, False alarm rate

\section{Introduction}

A wireless sensor network (WSN) has attracted considerable attention, because of its great potential in various applications such as battlefield surveillance, traffic, security, weather forecasts [1-3], health care, and home automation. Each sensor makes a local binary decision in a WSN that has $N$ distributed sensors and a fusion center (FC). Then, the sensors' decisions are sent to the FC to make a global decision that decides whether the target is present. Each sensor has a local threshold to make a decision about the target's presence or absence with or without the help of their neighbors' sensors.

Numerous papers have studied the conventional distributed detection problem. In [3, 4], the optimal fusion rule is derived under the conditional independence assumption. In [5], the authors develop a new and optimal algorithm for distributed detection in sensor networks over fading channels with multiple receiving antennas at the FC. It derives the optimal decision rules and the associated probabilities of detection and false alarm for three scenarios of channel

\footnotetext{
* Correspondence: liulg@126.com

${ }^{1}$ University of Electronic Science and Technology, Chengdu, Sichuan 611731, China

Full list of author information is available at the end of the article
}

state information availability. In [6], the authors propose a binary decision fusion scheme that reaches a global decision by integrating local decisions made by fusion members. Based on the minimax criterion, the optimal local thresholds and global threshold are derived without a preestimated target appearance probability. In [7], the authors develop a distributed detection approach based on recent development of the false discovery rate and the associated procedure by using the scalar test statistics. The local vote decision fusion algorithm, where sensors correct their decisions about the target's presence or absence using decisions of neighboring sensors, and then makes a collective decision, is proposed in [3]. An improved threshold approximation for local vote decision fusion is studied in [8]. The fusion threshold bounds derived in [9] using Chebyshev's inequality ensure a higher hit rate and lower false alarm rate without requiring a prior probability of target presence. In [10-14], the scan statistics is introduced to improve the detected performances. The performances of different approaches, the Chair-Varshney rule, generalized likelihood ratio test, and Bayesian view, are compared through simulations in [11]. Decision fusion with fading channels which are non-ideal channels is studied in $[5,15]$. The optimal power allocation between training and data at 
each sensor over orthogonal channels that are subject to path loss, Rayleigh fading, and Gaussian noise is derived in [16]. The authors design a computationally efficient fusion rule which involves injecting a deliberate random disturbance to the local sensor decisions before fusion in [17].

However, the traditional target detection can be seen as a binary classification problem which decides whether the target is present. The classification problem is widely studied in the field of machine learning (ML), pattern recognition, and statistical learning $[18,19]$. The learning problem can be classified into two classifications: supervised learning and unsupervised learning. In this paper, one is one standing for the target's presence, the other is zero standing for the target's absence. They have been known to us when we select training examples. Obviously, ML is used to solve the classical target detection which belongs to the field of supervised learning whose labels can be known.

In this paper, we first give the general formula of the $K$ out of $N(K / N)$ fusion rule, where $N$ is a variable. $K / N$ fusion rule is that the FC collects data from the local sensors which monitor the region of interest (ROI) and decides a target's presence when $k$ out of the data report "1." Then, the logistic regression fusion rule (LRFR) model derived to serve as the FC's fusion rule is introduced, and the logistic regression fusion algorithm (LRFA) is proposed. The counting rule and $K / N$ fusion rule are compared with the LRFR. Some numerical simulations are conducted and show their performances.
The rest of the paper is organized as follows. In Section 2, we introduce the signal decay model and basic assumptions. The local sensors' detection model is introduced with a WSN with distributed sensors and ideal communication channels between sensors and the fusion center. In Section 3, the fusion rules are given including the $K / N$ rule, the counting rule, and the LRFR. In Section 4, considering that the total number of sensors is fixed and is known to the FC, the LRFR is simulated. Then, when the variable number of sensors which send data to the FC is more than half of the total initially sensors, some simulations are conducted including LRFR and we compare the LRFR with the counting rule and the $K / N$ fusion rule. In Section 5, we summarize this paper.

\section{Sensor model}

Considering that a WSN with $N$ distributed sensors and a target, we assume that noise exists among the target and the sensors. The noise is independent and identically distributed (i.i.d) and follows the standard Gaussian distribution with zero mean and unit variance. As Fig. 1 shows, $N$ distributed sensors are uniformly deployed in the ROI which is a square with area A2, and a target randomly appears in the ROI. The locations of the local sensors are unknown to the $\mathrm{FC}$, and every local sensor first monitors the ROI, makes a local decision about the target's presence or absence, and then sends its decision to the FC to make a global decision which decides whether the target exists.

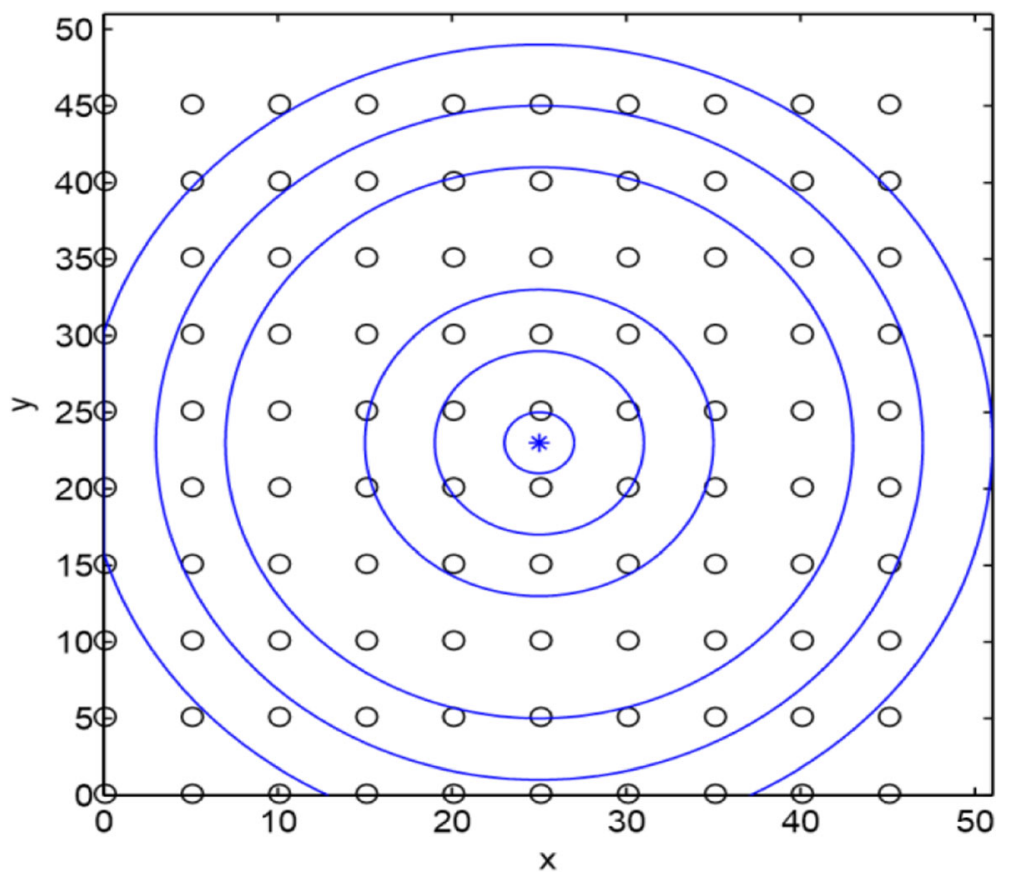

Fig. 1 A sensor deployment example. Area of the square region: A2; circle: the local sensors; star: the target 
For a local sensor $i$, the binary hypothesis testing problem is denoted as follows:

$$
\begin{aligned}
& H_{1}: r_{i}=s_{i}+n_{i} \\
& H_{0}: r_{i}=n_{i}
\end{aligned}
$$

where $H_{1}$ represents the hypothesis of the target's presence and $H_{0}$ represents the hypothesis of the target's absence. As discussed above, $n_{i}$ represents the noise and follows the standard Gaussian distribution

$$
n_{i} \sim N(0,1)
$$

In Eq. (1), $r_{i}$ denotes the signal received by the local sensor $i$, and $s_{i}$ denotes the target's signal detected by the local sensor $i$. The signal power emitted by the target decays as the distance from the target increases. The signal power attenuation model is as follows:

$$
s_{i}=g\left(d_{i}\right)
$$

where $d_{i}$ represents the distance between the target and the local sensor $i$ is denoted as

$$
d_{i}=\sqrt{\left(x_{i}-x_{t}\right)^{2}+\left(y_{i}-y_{t}\right)^{2}}
$$

and the function $g($.$) denotes the attenuation function$ with the distance from the target. In Eq. (4), $\left(x_{i}, y_{i}\right)$ represents the coordinate of the local sensor $i$ and $\left(x_{t}, y_{t}\right)$ represents the coordinate of the target. The function $g($.) has many different forms. One example for $g($.$) is de-$ noted in Eq. (21) as follows:

$$
g(x)=\left\{\begin{array}{cc}
P_{0} & x<d_{0} \\
P_{0} / x_{x^{\beta}} & x \geq d_{0}
\end{array}\right.
$$

where $p_{0}$ denotes the signal power emitted by the target at a reference distance $d_{0}$, and $\beta$ is the signal decay exponent. In Eq. (22), the function $g($.$) which is used in$ this paper is given as follows:

$$
g\left(d_{i}\right)=\sqrt{\frac{P_{0}}{1+\lambda d_{i}^{\beta}}}
$$

where $\lambda$ is an adjustable constant and $\beta$ often takes values between 2 and 3 . Assuming that the local sensor $i$ uses the threshold $\tau_{i}$ to make the local binary decision $I_{i}$, in which $I$ denotes that the target is present and 0 denotes that the target is absent, then all decisions denoted by $I=\left(I_{1}, I_{2}\right.$, $\left.\ldots, I_{N}\right)$ are sent to the FC to make a global decision that 1 represents the target's presence and 0 presents the target's absence. According to the Neyman-Pearson (NP) lemma, the local sensor-level false alarm rate and probability of detection are given by

$$
P_{f i}=P\left(I_{i} \mid H_{0}\right)=Q\left(\tau_{i}\right)
$$

$$
P_{d i}=P\left(I_{i} \mid H_{1}\right)=Q\left(\tau_{i}-s_{i}\right)
$$

where $Q(x)=\int_{x}^{\infty} \frac{1}{\sqrt{2 \pi}} e^{-\frac{y^{2}}{2}} d y$, which is the complementary distribution function of the standard Gaussian.

In WSNs for distribution detection, the local observation has to be quantized before being transmitted to the $\mathrm{FC}$ which is demanded to make the final decision about the target's presence.

\section{Fusion rules}

In this paper, we assume that all the local sensors have the same false alarm rate, that is, they have the same local threshold to make a local decision (Eqs. (21) and (22)). The local false alarm rate satisfies Eq. (7). We also assume that the wireless channels between the sensors and the fusion center are perfect, with negligible error rates. Three fusion rules will be shown in this section.

\section{1 $\mathrm{K} / \mathrm{N}$ fusion rule}

$K / N$, which is short for $K$ out of $N$, is that the FC selects the data from the local sensors and makes a positive decision when more than $k$ data values are " 1 "s. The formula of $K / N$ fusion rule is given by

$$
A_{1}=\sum_{i=1}^{N} I_{i} \underset{H_{0}}{\stackrel{H_{1}}{\longrightarrow}} K
$$

where $N$ is the total number of the sensors. When the FC receives the variable number of sensors, Eq. (9) can be written as

$$
A_{2}=\frac{\sum_{i=1}^{N} I_{i}}{m} \underset{H_{0}}{\stackrel{H_{1}}{\geq}} k
$$

where $m$ denotes that the total number of sensors which send data to the FC at the moment and $k \in(0,1), m \leq N$.

\subsection{Counting rule}

The counting rule, which is widely used as the fusion rule of the FC in Eqs. (6), (10), (22), and (23), is an intuitive choice that is to use the total number of " 1 " as a statistic. The counting rule makes a global decision by first counting the number of detections made by the local sensors and then comparing it with a threshold $T$. The counting rule can be written as

$$
A_{3}=\sum_{i=1}^{N} I_{i} \underset{H_{0}}{\stackrel{H_{1}}{\gtrless}} T
$$

where $N$ is the total number of the sensors which send data to the FC and $T$ is a threshold. The K/Nis the uniting rule when $T$ is a constant. The global false alarm rate can be given as 


$$
P_{\mathrm{Fa}}=P_{r}\left\{\Lambda 3 \geq T \mid H_{0}\right\}
$$

where $P_{\mathrm{Fa}}$ is the global false alarm rate. When every local sensor has the same false alarm rate, the global false alarm rate can be written as

$$
P_{\mathrm{Fa}}=\sum_{i=T}^{N}\left(\begin{array}{c}
N \\
i
\end{array}\right) p_{f i}^{i}\left(1-p_{f i}\right)^{N}
$$

When $N$ is large enough, Eq. (13) can be obtained by using the Laplace-DeMoivre approximation

$$
P_{\mathrm{Fa}}=Q\left(\frac{T-N p_{f i}}{\sqrt{N p_{f i}\left(1-p_{f i}\right)}}\right)
$$

The threshold can be calculated as Eq. (22):

$$
T=Q^{-1}\left(P_{\mathrm{Fa}}\right) \sqrt{N p_{f i}\left(1-p_{f i}\right)}+N p_{f i}
$$

From Eq. (15), it is clear that $T$ is a function of the global false alarm rate, the local sensors' false alarm rate, and the total number of the sensors which send data to the FC.

\subsection{LRFR}

The LRFR uses the logistic regression to train the weights to make a decision. The logistic regression can be expressed as

$$
h(x)=\frac{1}{1+\exp \left(-W^{T} X\right)}
$$

where $W$ is the weight vector, and $x$ is the feature. $x$ can include the total number of the sensors which send data to the $\mathrm{FC}$, the number of " 1 " reported by the sensors to the
FC, and the local sensors' performances (such as false alarm rate, detection probabilities), and the constant "1," etc.

$N$ denotes the total number of the sensors which send data to the FC. The equation $m=N \sum_{i=1}^{m} I_{i}$ denotes the number of "1" reported by the local sensors. The red star means the target is present, and the black cross means the target is absent. $A=50, P_{0}=1500, \tau=1.6449, \lambda=$ $1, \beta=2, N=100$.

As shown in Fig. 2, 10,000 simulations are conducted. The total number of the local sensors is 100 and fixed, that is, the FC selects data from the local sensors and makes a decision about the target's presence when the data are equal to 100 at that moment. Some important parameters are set as follows: the area of ROI is 50, the power of the target is 1500 , and the local sensors' threshold is 1.6449. The red star denotes that the real circumstance has the target. The black cross in Fig. 2 denotes that the real circumstance does not have the target. From Fig. 2, we can see that it has a clearly separable region between the target's presence and the target's absence.

Ten thousand simulations are conducted in Fig. 3, where $m$ is a variable that denotes the sensors' sent data to the FC at the moment. We assume that the FC makes a decision when $m$ is equal to or more than half of the initially total number of the local sensors at that moment. Some important parameters are set as follows: the area of $\mathrm{ROI}$ is 50 , the power of the target is 1500 , and the local sensors threshold is 1.6449. The red star in Fig. 3 represents the target's presence and the black cross represents the target's absence. As shown in Fig. 3, it also has a clearly separable region between the target's presence and the target's absence.

So, when $N$ is known to the FC and $m=N$, the parameters of the logistic regression can be set as follows:

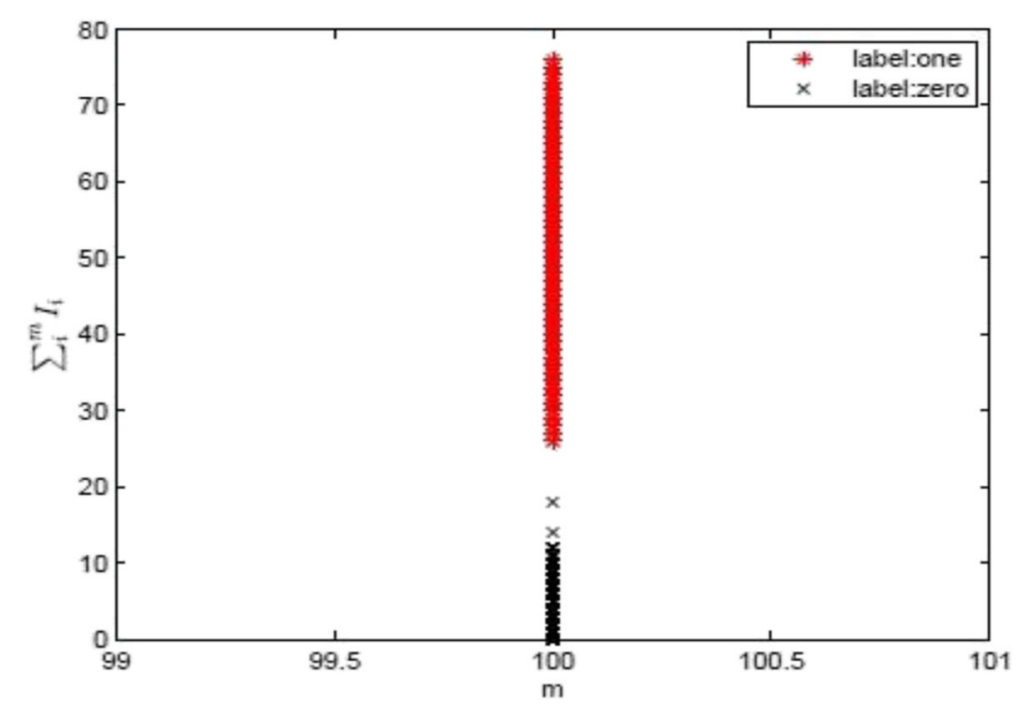

Fig. 2 The FC selects data from the local sensors 


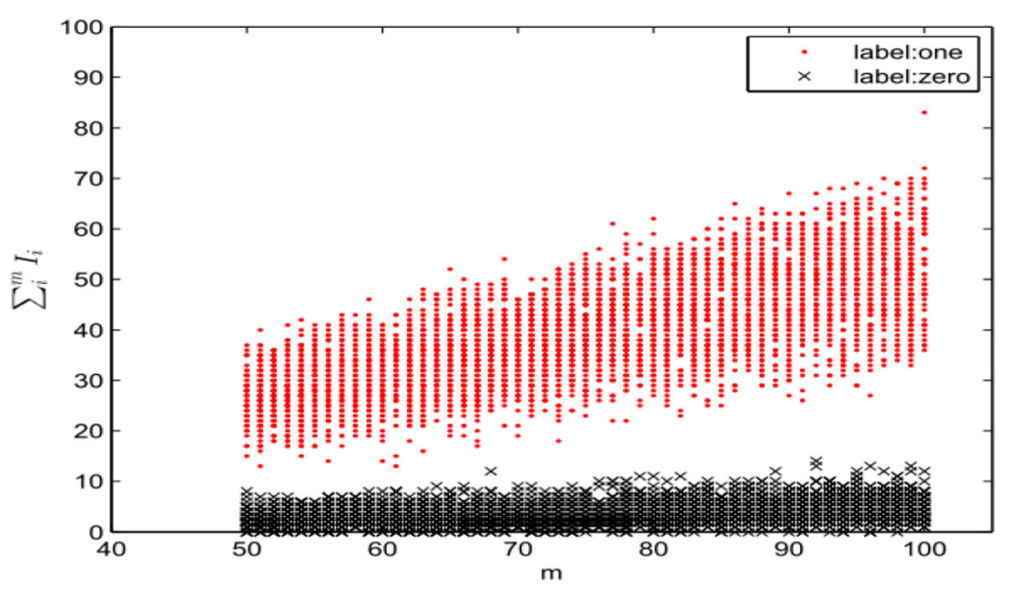

Fig. 3 The FC selects data from the local sensors

$$
\begin{gathered}
w=\left[\begin{array}{ll}
w 1 & w 0
\end{array}\right] \\
x=\left[\begin{array}{ll}
\sum_{i=l}^{m} I_{i} & 1
\end{array}\right]
\end{gathered}
$$

where $m$ is a variable and more than or equal to half of $N$. $\sum_{i=1}^{m} I_{i}$ denotes the number of " 1 " reported by the local sensors. The red star means the target is present, and the black cross means the target is absent. $A=50, P_{0}$ $=1500, \tau=1.6449, \lambda=1, \beta=2, N=100 . \quad \sum_{i=1}^{m} I_{i}$ denotes the number of " 1 " reported to the FC by the local sensors at that moment. When $m$ is a variable, the parameters of the logistic regression can be obtained by

$$
\begin{aligned}
& w=\left[\begin{array}{lll}
w 1 & w 2 & w 0
\end{array}\right] \\
& x=\left[\begin{array}{lll}
m \sum_{i=l}^{m} I_{i} & 1
\end{array}\right]
\end{aligned}
$$

The LRFR can be obtained by

$\Lambda_{4}=\operatorname{sign}(h(x)-\varphi) \underset{H_{0}}{\stackrel{H_{1}}{\gtrless} 0}$

where $\operatorname{sign}(x)$ is the sign function, $h(x)$ stands for Eq. (16), and $\varphi$ is a constant. The miss alarm rate rises with increasing $\varphi$, and the false alarm rate rises with decreasing $\varphi$. Let $\varphi=0.5$, we have $W^{T} x=0$. So, Eq. (19) can be simplified as

$$
\Lambda 5=\operatorname{sign}(x) 0_{H 0}^{H 1}
$$

\subsection{LRFA}

One of the LRFR's most important steps is to obtain the parameters. To solve the problem, we can get the parameters by the maximum likelihood estimator. Let

$$
\begin{aligned}
& P(u=1 \mid x ; w)=h(x) \\
& P(u=0 \mid x ; w)=1-h(x)
\end{aligned}
$$

where $u$ denotes the FC's decision, $x$ denotes the input of the features, $w$ is the weight of the LRFR, and $h(x)$ represents Eq. (16). Because of the fact that $u$ can only be 1 or 0 , the likelihood function can be expressed by

$$
P(u \mid x ; w)=h(x) u(1-h(x)) 1-u
$$

With the independent training samples, the likelihood function can be written as

$$
L(\mathbf{w})=P(\mathbf{u} \mid \mathbf{x} ; \mathbf{w})=\prod_{i}^{M} P\left(u_{i} \mid \mathbf{x}_{\mathbf{i}} ; \mathbf{w}\right)=\prod_{i}^{M} h\left(\mathbf{x}_{i}\right)^{u_{i}}\left(1-h\left(\mathbf{x}_{i}\right)\right)^{1-u_{i}}
$$

where $M$ is the number of the training samples. It will be easier to maximize the log likelihood

$$
\begin{aligned}
l(\mathbf{w}) & =\log \{\mathbf{L}(\mathbf{w})\} \\
& =\sum_{i}^{M}\left\{u_{i} \log h\left(\mathbf{x}_{i}\right)+\left(1-u_{i}\right) \log \left(1-h\left(\mathbf{x}_{i}\right)\right)\right\}
\end{aligned}
$$

To solve the parameters of the LRFR, penalized logistic regression is often used in ML. The general formula is as follows:

$$
-l(\mathbf{w})+p(\mathbf{w})=-\sum_{i}^{M}\left\{u_{i} \log h\left(\mathbf{x}_{i}\right)+1\left(1-u_{i}\right) \log \left(1-h\left(\mathbf{x}_{i}\right)\right)\right\}+p(\mathbf{w})
$$

where $P(w)$ is the penalized function. To minimize Eq. (26), we can use the gradient descent. In Eq. (24), as an optimization problem, binary classification $L 2$ penalized logistic regression minimizes the following cost function

$$
\min \left\{\frac{1}{2} \mathbf{w}^{T} \mathbf{w}-C \sum_{i}^{M}\left\{u_{i} \log h\left(\mathbf{x}_{i}\right)+\left(1-u_{i}\right) \log \left(1-h\left(\mathbf{x}_{i}\right)\right)\right\}\right\}
$$

where $C$ controls the level of regularization. 


\section{Algorithm 1 LRFA}

1: Set the $p_{f i}$;

2: Generate the training data by conducting the following experiments:

1) Set the target's presence;

2) Select the data into $x$ in Eq. (17) or Eq. (18);

3) Set the target's absence;

4) Select the data into $x$ in Eq. (17) or Eq. (18);

5) Repeat $n$ times from 1) to 4).

3: Train the LRFR with the data set by Eq. (27);

4: Train the LRFR with the data set by Eq. (28);

5: Compare the LRFR between L1 and $L 2$ regularized logistic regression, and select a proper LRFR;

6: Use the LRFR as the $F C$ 's fusion rule.

The LRFA is shown as pseudo-code in Algorithm 1. Step 1 is only in numerical simulations, that is, the LRFR does not need the knowledge of the performances of local sensors. Steps 2 and 4 select Eq. (17) or Eq. (18) with the dependence of whether the total number of the sensors which send data to the FC is variable. Steps 3 and 4 train the LRFR with L1 and L2, respectively. The performances of the LRFR with L1 and L2 are computed, and a proper LRFR (step 4 in Algorithm 1) is selected. Through the LRFA, a proper LRFR is selected as the fusion rule of the FC.

$\sum_{i=1}^{m} I_{i}$ denotes the number of " 1 " reported by the local sensors, where $m=N$. The red star means the target is present, and the black cross means the target is absent. $A=50, P_{0}=1500, \tau=1.6449, \lambda=1, \beta=2, N=100$.

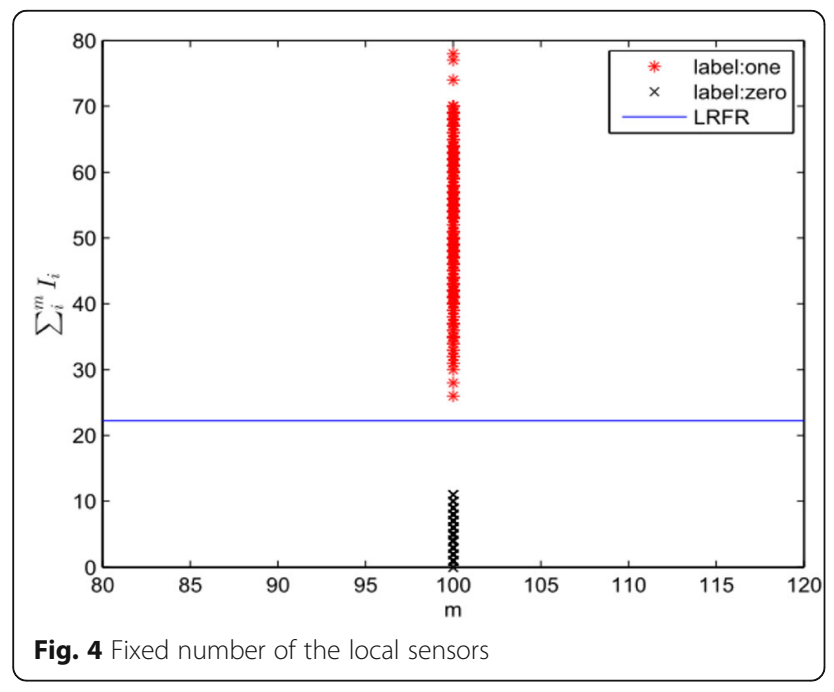

The $m$ is a variable and more than or equal to half of $N$. $\sum_{i=1}^{m} I_{i}$ denotes the number of " 1 " reported by the local sensors. The red star means the target is present, and the black cross means the target is absent. $A=50$, $P_{0}=1500, \tau=1.6449, \lambda=1, \beta=2, N=100$.

\section{Numerical simulations}

In this section, we conduct numerical simulations. The software of the numerical simulations used in this section are Matlab and Python. One of the packages of the Python is scikit-learn (Eq. (24)). Scikit-learn is on ML in Python, which is a simple and efficient tool for data mining and data analysis. It includes classification, regression, clustering, dimensionality reduction, model selection, and reprocessing.

As shown in Fig. 4, 1000 training samples are used to train the weights of Eq. (17). Some important parameters

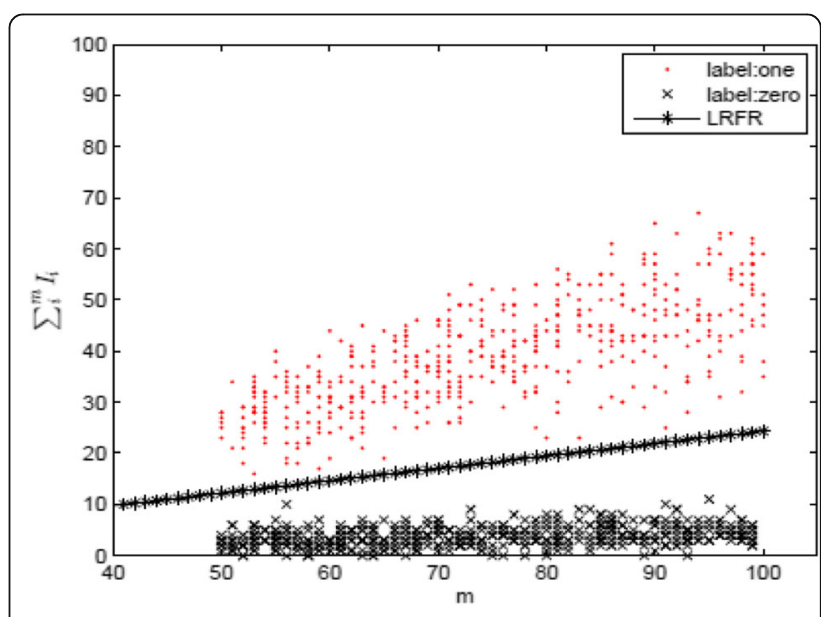

Fig. 5 The variable number of local sensors 


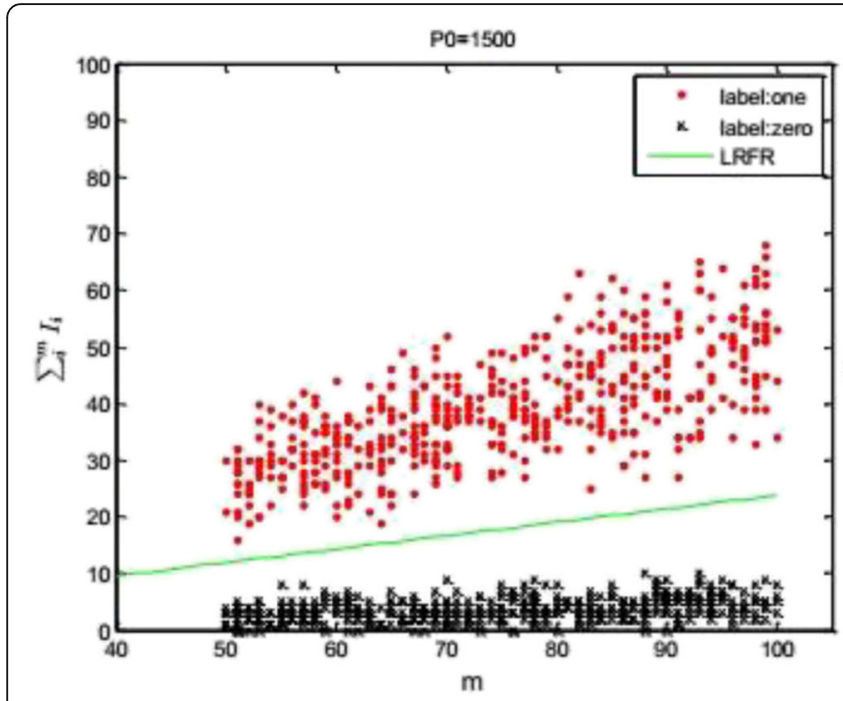

a

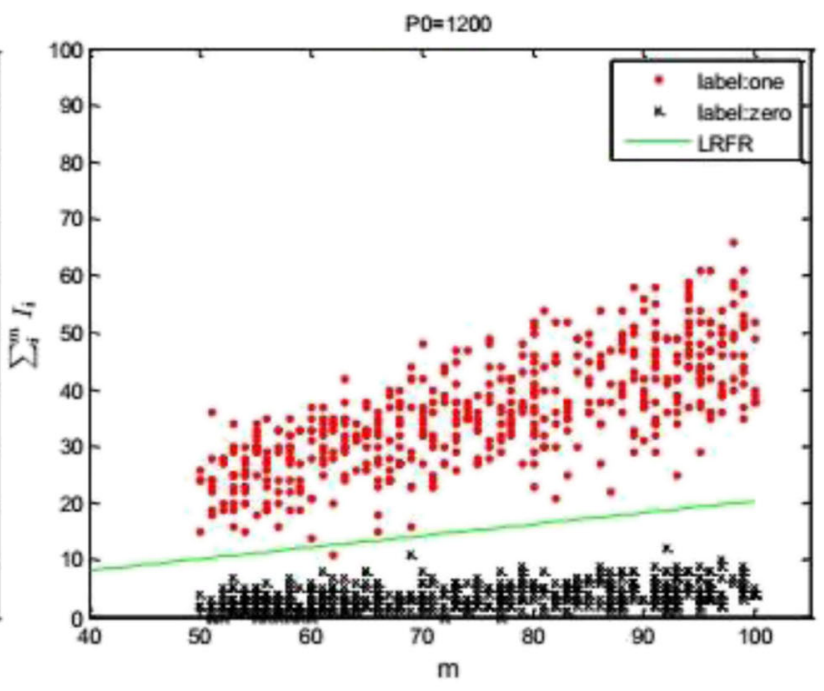

b

Fig. 6 The variable total local sensors. The $m$ is a variable and more than or equal to half of $N$. $\sum_{i}^{m} l_{i}$ denotes the number of "1" reported by the local sensor. The red star means the target is present, and the black cross means the target is absent. $A=50, \tau=1.6449, \lambda=1, \beta=2, N=100$. a $P_{0}=1500$. b $P_{0}=900$

are set as follows: $A=50, P_{0}=1500, \tau=1.6449, \lambda=1, \beta=$ $2, N=100$. The number of the local sensors which send their data to the FC is fixed, that is, the FC makes a decision about the target's presence when detections sent to the FC are up to $N$. We get the weights of Eq. (17) by the LRFA, and we generate 1000 random samples to test. From Fig. 4, the two labels of test samples can be clearly separated.

To show the influences of the variable total number of the local sensors which send data to the FC at that moment, in Fig. 5, we assume that the FC makes decisions when the number of detections sent to the FC at that moment is equal to or more than half of the initially total number of sensors deployed in ROI. The weights of Eq. (18) are trained by 1000 training samples. Some important parameters are set: $A=50, P_{0}=1500, \tau=1.6449, \lambda=1, \beta=$ $2, N=100$. In this simulation, we can see the LRFR clearly separate the two labels (in Fig. 5).

To see the influences of the different power of the target, we conduct variable powered experiments. The power of the target varies from 1500 to 100 . The weights of Eq. (18) are solved by 10,000 training samples. Some important parameters are set: $A=50, \tau=1.6449, \lambda=1, \beta=2, N=100$, $L=L 2$. From Fig. 6 , the region between the two labels becomes small with the decrease of the target power. From

Table 1 The performances of the system with varying power

\begin{tabular}{lllllll}
\hline$P_{0}$ & 1500 & 1200 & 900 & 600 & 300 & 100 \\
$P_{\mathrm{D}}$ & $99.96 \%$ & $99.96 \%$ & $99.94 \%$ & $99.80 \%$ & $99.10 \%$ & $94.72 \%$ \\
$P_{\mathrm{Fa}}$ & $0 \%$ & $0 \%$ & $0 \%$ & $0.18 \%$ & $0.92 \%$ & $9.60 \%$ \\
\hline
\end{tabular}

Eqs. (1) and (6), we know the received signal of the local sensors decreases with the decreasing power. The weights of Eq. (18) are solved by the 10,000 training samples. The LRFR can classify the target's presence and absence with 1000 random test samples. Table 1 shows the performances of the FC change with the power of the target. The detected probability of the FC becomes small, and the false alarm rate of the FC becomes large with the power decreasing.

Figure 7 shows the comparison between LRFR and $K /$ $N$. Some important parameters are set: $A=50, P_{0}=1500$, $\tau=1.6449, \lambda=1, \beta=2, N=100$. The LRFR can have the same ability of the $k / N$. However, there are no good

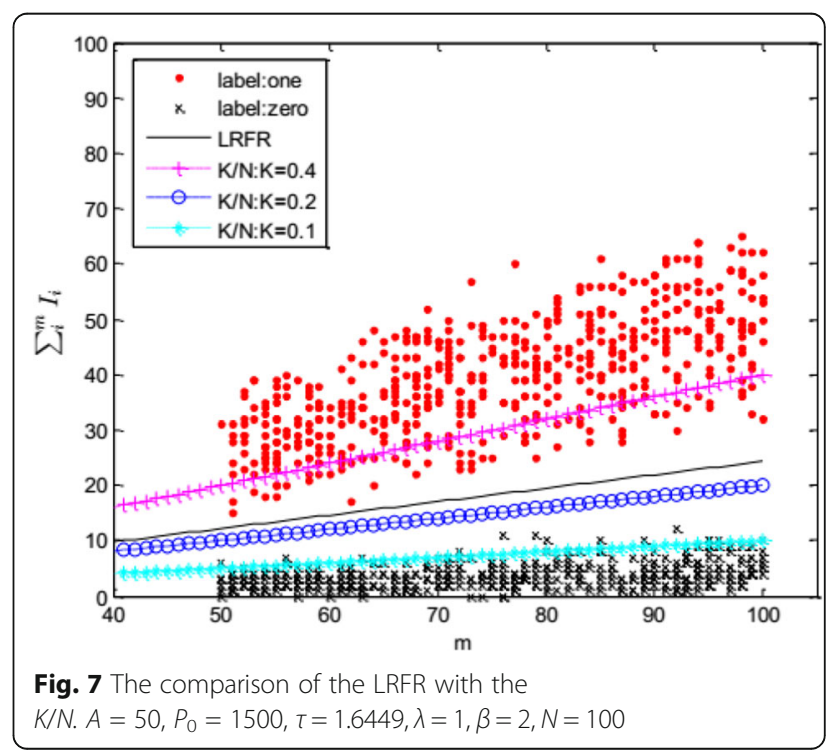




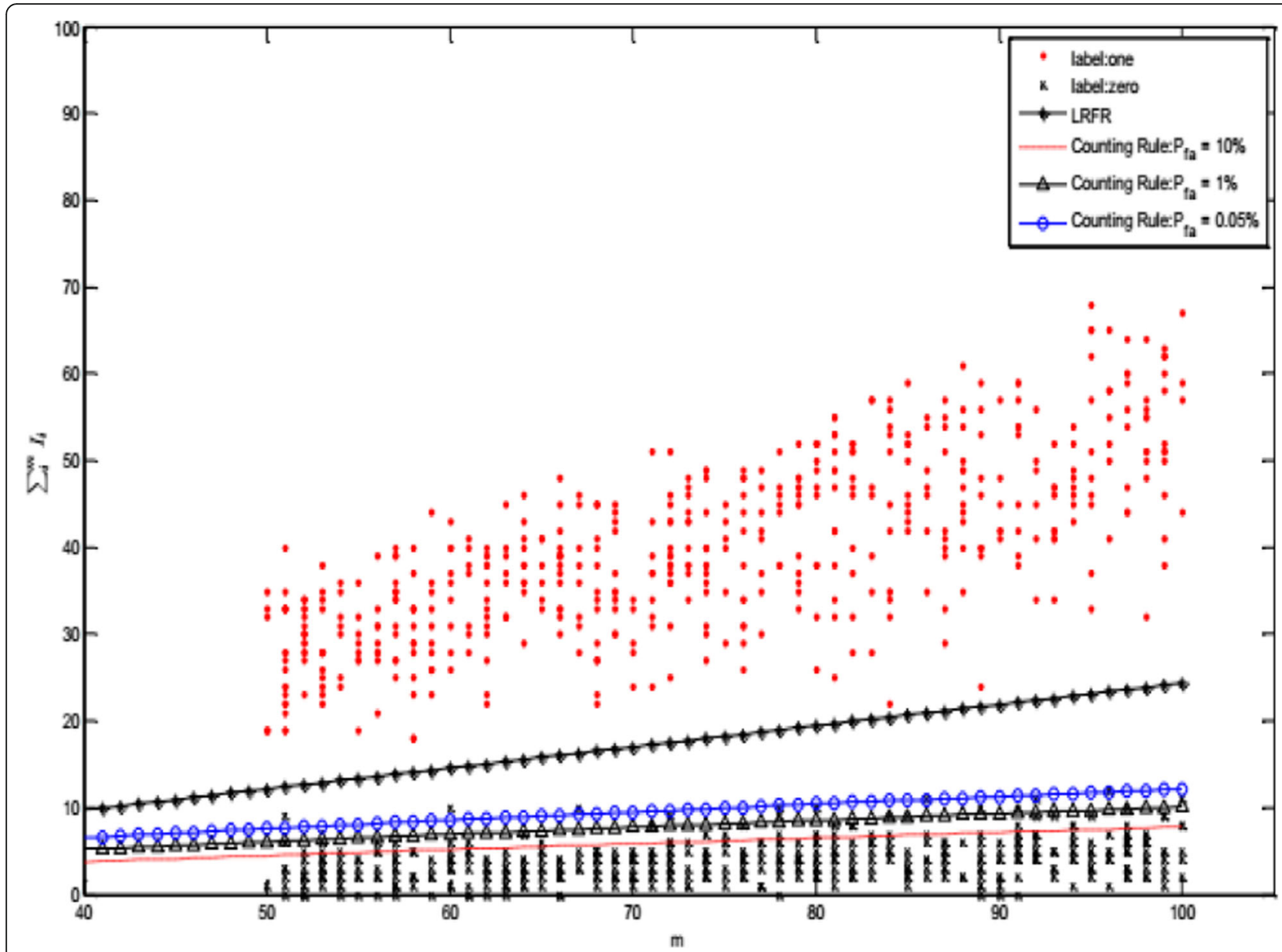

Fig. 8 The comparison of the LRFR with the counting rule. $A=50, P_{0}=1500, \tau=1.6449, \lambda=1, \beta=2, N=100$

methods to solve the parameter $k$ of the $K / N$ fusion rule. The LRFR can be easily solved by the ML method. To study the difference between the LRFR and the counting rule, in Fig. 8, the parameters are the same as Fig. 7. The performances of counting rule become decreasing with the global increasing false alarm rate. From Eq. (15), we know that the performances of the counting rule have the relation with the global false alarm rate.

\section{Conclusions}

In this paper, we give the general formula of the $k / N$ fusion rule based on the total number of detections made by the local sensors. The LRFR and LRFA are proposed. The fixed total number of detections and the variable number of detections made by the local sensors are considered. The numerical simulations are given as above. From the numerical simulations, we see the LRFR can be well used as the FC's fusion rule because of the outstanding performances and low computational complexity. One of the drawbacks of the LRFR is that it requires training samples to solve the weights of the LRFR. In the future, different methods of the ML will be studied to solve the problems of the target detection and different features will be considered.

\section{Authors' information}

Longgeng Liu (b. June 5, 1975) received his M.Sc. in Computer Science (2000) from a university. Now, he is a director at China National Software and Integrated Circuit Promotion Center of the Ministry of Industry and Information Technology. He is a Ph.D. and studies at the University of Electronic Science and Technology. His current research interests include different aspects of artificial intelligence and distributed systems. He has (co-)authored more than two books and ten papers.

\section{Competing interest}

The authors declare that they have no competing interests.

\section{Author details}

'University of Electronic Science and Technology, Chengdu, Sichuan 611731, China. ${ }^{2}$ Chongqing University of Posts and Telecommunications, Chongqing 400065, China.

Received: 23 May 2016 Accepted: 12 December 2016

Published online: 07 January 2017

\section{References}

1. C. Fonseca, H. Ferreira, Stability and contagion measures for spatial extreme value analyses. arXiv. 1206-1228 (2012) 
2. K Athanasios, Z Wang, A Rodrłguez, Spatial modeling for risk assessment of extreme values from environmental time series: a Bayesian nonparametric approach. Environmetrics 23(8), 649-662 (2012)

3. N Katenka, E Levina, G Michailidis, Local vote decision fusion for target detection in wireless sensor networks. IEEE Trans. Signal. Process. ACM 56(1), 329-338 (2008)

4. P. K. Varshney, Distributed detection and data fusion. New York: SpringerVerlag New York, Inc. 36-118 (1996)

5. I. Nevat, G. Peters, I. Collings, Distributed detection in sensor networks over fading channels with multiple antennas at the fusion center. 1(3), 45-49 (2014)

6. SH Javadi, A Peiravi, Fusion of weighted decisions in wireless sensor networks. Wireless Sens. Syst. IET 5(2), 97-105 (2015)

7. E Ermis, S Venkatesh, Distributed detection in sensor networks with limited range multimodal sensors. Signal. Process. IEEE Trans. 58(2), 843-858 (2010)

8. Ridout, S Martin, An improved threshold approximation for local vote decision fusion. Signal. Process. IEEE Trans. 61(5), 1104-1106 (2013)

9. M Zhu et al., Fusion of threshold rules for target detection in wireless sensor networks. ACM Trans. Sens. Netw. (TOSN) 6(2), 18 (2010)

10. X Song et al., Active detection with a barrier sensor network using a scan statistic. IEEE J. Ocean. Eng. 37(1), 66-74 (2012)

11. M Guerriero, S Lennart, W Peter, Bayesian data fusion for distributed target detection in sensor networks. Signal. Process. IEEE Trans. 58(6), 3417-3421 (2010)

12. M Guerriero, W Peter, G Joseph, Distributed target detection in sensor networks using scan statistics. Signal. Process. IEEE Trans. 57(7), 2629-2639 (2009)

13. J Glaz, Z Zhang, Multiple window discrete scan statistics. J. Appl. Stat. 31(8), 967-980 (2004)

14. J Glaz, NI Naus, Tight bounds and approximations for scan statistic probabilities for discrete data. Ann. Appl. Probab. 1(2), 306 (1991)

15. B Chen et al., Channel aware decision fusion in wireless sensor networks. Signal. Process. IEEE Trans. 52(12), 3454-3458 (2004)

16. R Hamid, V Azadeh, Optimal training and data power allocation in distributed detection with inhomogeneous sensors. Signal Processing Letters. IEEE. 20(4), 339-342 (2013)

17. G Satish, R Niu, K Pramod, Fusing dependent decisions for hypothesis testing with heterogeneous sensors. Signal. Process. IEEE Trans. 60(9), 4888-4897 (2012)

18. T Hastie et al., The elements of statistical learning: data mining, inference, and prediction. 2 (Springer, New York, 2009), pp. 65-104

19. C.M. Bishop, Pattern recognition and machine learning information science and statistics Secaucus. 2(3), 045-108 (2006)

\section{Submit your manuscript to a SpringerOpen ${ }^{\circ}$ journal and benefit from:}

- Convenient online submission

- Rigorous peer review

- Immediate publication on acceptance

- Open access: articles freely available online

- High visibility within the field

Retaining the copyright to your article 